\title{
The Delay Effect in a Stochastic Multiplier-Accelerator Model
}

\section{Ioannis K. Dassios • Alexandros A. Zimbidis • Charalambos P. Kontzalis}

Received: 26 March 2014 / Revised: 23 August 2014 / Accepted: 11 November 2014 /

Published online: 27 November 2014

(C) 2014 Dassios et al.; licensee Springer. This is an Open Access article distributed under the terms of the Creative Commons Attribution License (http://creativecommons.org/licenses/by/4.0), which permits unrestricted use, distribution, and reproduction in any medium, provided the original work is properly credited.

\begin{abstract}
This paper extends the classical Samuelson multiplier-accelerator model for national economy. Actually, this new modeling structure removes the basic shortcoming of the original model producing stable business cycles when realistic values of the parameters (multiplier, accelerator) are entered into the system of equations. Under this new approach, we introduce some kind of randomness and memory into the system. We assume that consumption, private investment and governmental expenditure depend upon the national income values of the last $n(n>1)$ years and further assume that multiplier and accelerator factors are stochastic variables. Then stochastic delayed difference equations of higher order are employed to describe the model, while the respective solutions of higher order polynomials for the expectation of national income variables correspond to the typical observed business cycles of real economy. Stability and controllability conditions are investigated while numerical examples provide further insight and better understanding as regards the control actions, system design, and produced business cycles.
\end{abstract}

\footnotetext{
I.K. Dassios

MACSI, Department of Mathematics \& Statistics, University of Limerick, Limerick, Ireland e-mail: jdasios@math.uoa.gr
}

I.K. Dassios

ERC, Electricity Research Centre, University College Dublin, Dublin, Ireland

\author{
A.A. Zimbidis \\ Athens University of Economics and Business, 76 Patission Str., Athens, 15784, Greece \\ C.P. Kontzalis $(\bowtie)$ \\ Department of Informatics, Ionian University, Corfu, Greece \\ e-mail: kontzalis@ath.forthnet.gr \\ C.P. Kontzalis \\ Basel Area, SBB, Swiss Federal Railways, Basel, Switzerland
}


Keywords Samuelson model $\cdot$ Stochastic $\cdot$ Difference equations · Stability · Control · State feedback

\section{Introduction}

Keynesian macroeconomics inspired the seminal work of Samuelson (1939), who actually introduced the business cycle theory. Although primitive and using only the demand point of view, Samuelson's prospect still provides an excellent insight into the problem and justification of business cycles appearing in national economies. In the past decades, many more sophisticated models have been proposed by other researchers; see Chari (1994), Chow (1985), Dassios and Kontzalis (2012), Dassios et al. (2012), Dassios and Kalogeropoulos (2014), Dassios and Zimbidis (2014), Day (1999), Puu et al. (2004), Rosser (2000), Westerhoff (2006), Wincoop (1996). All these models use superior and more delicate mechanisms involving monetary aspects, inventory issues, business expectation, borrowing constraints, welfare gains, and multi-country consumption correlations.

Some of the previous articles also contribute to the discussion for the inadequacies of Samuelson's model. The basic shortcoming of the original model is: the incapability to produce a stable path for the national income when realistic values for the different parameters (multiplier and accelerator parameters) are entered into the system of equations. Of course, this statement contradicts with the empirical evidence which supports temporary or long-lasting business cycles.

In this article, we propose a generalization of the typical model incorporating delayed variables into the system of equations. The proposed modification succeeds to provide a more comprehensive explanation for the emergence of business cycles while also produce a stable trajectory for the expectation of the national income. Actually, it succeeds to model stable business cycles when realistic and stochastic values of the multiplier and accelerator parameters are entered into the system of equations. So, we can safely deduce the fact that individuals and/or institutions base their decisions (for consumption or investment levels) upon the experience of the last $n$ years, $n>1$.

The rest of this paper is organized as follows. Section 2 contains three subsections: Sect. 2.1 provides a short review for the organization of the original model, Sect. 2.2 discusses the incentives of a reformulation based on delayed information structure and also providing the system of difference equations governing the whole model and Sect. 2.3 provides a practical justification for the reformulated model. Section 3 investigates the stability of some interesting special cases. Section 4 suggests a typical state feedback action for the different parameters involved and also proposes how to design the corresponding solution trajectories. Section 5 contains some numerical examples and Sect. 6 concludes the entire paper.

\section{The Model}

\subsection{Samuelson's Original Multiplier-Accelerator Model}

The original version of Samuelson's model is based on the following assumptions: 
Assumption 2.1 National income $T_{k}$ in year $k$, equals the summation of three elements: consumption, $C_{k}$, private investment, $I_{k}$, and governmental expenditure $G_{k}$

$$
T_{k}=C_{k}+I_{k}+G_{k}
$$

Assumption 2.2 Consumption $C_{k}$ in year $k$, depends on past income (only on last year's value) and on marginal tendency to consume, modeled with $a$, the multiplier parameter, where $0<a<1$,

$$
C_{k}=a T_{k-1}
$$

Assumption 2.3 Private investment $I_{k}$ in year $k$, depends on consumption changes and on the accelerator factor $b$, where $b>0$. Consequently, $I_{k}$ depends on national income changes,

$$
I_{k}=b\left(C_{k}-C_{k-1}\right)=a b\left(T_{k-1}-T_{k-2}\right) .
$$

Assumption 2.4 Governmental expenditure $G_{k}$ in year $k$, remains constant

$$
G_{k}=\bar{G}
$$

Hence, the national income is determined via the following second-order linear difference equation:

$$
T_{k+2}-a(1+b) T_{k+1}+a b T_{k}=\bar{G} .
$$

\subsection{The Reformulation-Delayed Samuelson's Model}

The reformulated (delayed) version of Samuelson's model is based on the following assumptions:

Assumption 2.5 National income $T_{k}$ in year $k$, equals the summation of three elements: consumption, $C_{k}$, private investment, $I_{k}$, and governmental expenditure $G_{k}$ :

$$
T_{k}=C_{k}+I_{k}+G_{k}
$$

Assumption 2.6 Consumption $C_{k}$ in year $k$, depends on past income (on more than one past year's value) and on marginal tendencies to consume, modeled with $a_{1}, a_{2}, \ldots, a_{n}$, the multiplier parameters, where $0<a_{1}+a_{2}+\cdots+a_{n}<1$,

$$
C_{k}=a_{1} T_{k-1}+a_{2} T_{k-2}+\cdots+a_{n} T_{k-n} .
$$

Assumption 2.7 Private investment $I_{k}$ in year $k$, depends on consumption changes and on the positive accelerator factors $b_{1}, b_{2}, \ldots, b_{m}$. Consequently, $I_{k}$ depends on the respective national income changes,

$$
I_{k}=b_{1}\left(C_{k}-C_{k-1}\right)+b_{2}\left(C_{k-1}-C_{k-2}\right)+\cdots+b_{m}\left(C_{k-m+1}-C_{k-m}\right) .
$$


By using Eq. (2) we get

$$
\begin{aligned}
I_{k}= & b_{1}\left(\sum_{i=1}^{n} a_{i} T_{k-i}-\sum_{i=1}^{n} a_{i} T_{k-(i+1)}\right)+\cdots \\
& +b_{m}\left(\sum_{i=1}^{n} a_{i} T_{k-(i+m-1)}-\sum_{i=1}^{n} a_{i} T_{k-(i+m)}\right),
\end{aligned}
$$

or, equivalently,

$$
I_{k}=\sum_{j=1}^{m} b_{j}\left(\sum_{i=1}^{n} a_{i} T_{k-(i+j-1)}-\sum_{i=1}^{n} a_{i} T_{k-(i+j)}\right) .
$$

Assumption 2.8 Governmental expenditure $G_{k}$ in year $k$, obeys the feedback law $I_{k}$ in year $k$, depends on consumption changes and on the positive accelerator factors $c_{1}, c_{2}, \ldots, c_{p}$. Consequently, $G_{k}$ is equal to,

$$
G_{k}=\bar{G}_{k}+c_{1} T_{k-1}+c_{2} T_{k-2}+\cdots+c_{p} T_{k-p} .
$$

Hence, by replacing Eqs. (2), (3), (4) into Eq. (1), the national income is determined via the following high-order linear difference equation:

$$
\begin{aligned}
T_{k}= & \bar{G}_{k}+\sum_{i=1}^{p} c_{i} T_{k-i}+\sum_{i=1}^{n} a_{i} T_{k-i} \\
& +\sum_{j=1}^{m} b_{j}\left(\sum_{i=1}^{n} a_{i} T_{k-(i+j-1)}-\sum_{i=1}^{n} a_{i} T_{k-(i+j)}\right) .
\end{aligned}
$$

Here $\bar{G}_{k}$, may be a fully controlled item by the government.

Assumption 2.9 The multiplier and accelerator parameters follow stochastic patterns. They are actually random variables where

$$
\begin{aligned}
& \mathbb{E}\left[A_{i, k}\right]=a_{i}, \quad i=1,2, \ldots, n, \\
& \mathbb{E}\left[B_{i, k}\right]=b_{i}, \quad i=1,2, \ldots, m .
\end{aligned}
$$

We use the basic equation for conditional expectations, i.e.

$$
E(X)=E(E[X \mid Y]),
$$

where $X, Y$ are random variables. Additionally, we define the information filtration $\mathcal{H}_{k}$ as all the events prior and inclusive of time $k$. Then, according to relationship (6) and by using Eq. (5), we obtain

$$
\tau_{k}=E\left[T_{k}\right]=E\left[E\left[T_{k} \mid \mathcal{H}_{k}\right]\right],
$$


or, equivalently,

$$
\begin{aligned}
\tau_{k}= & \bar{G}_{k}+\sum_{i=1}^{p} c_{i} E\left[T_{k-i}\right]+\sum_{i=1}^{n} a_{i} E\left[T_{k-i}\right] \\
& +\sum_{j=1}^{m} b_{j}\left(\sum_{i=1}^{n} a_{i} E\left[T_{k-(i+j-1)}\right]-\sum_{i=1}^{n} a_{i} E\left[T_{k-(i+j)}\right]\right),
\end{aligned}
$$

or, equivalently,

$$
\begin{aligned}
\tau_{k}= & \bar{G}_{k}+\sum_{i=1}^{p} c_{i} \tau_{k-i}+\sum_{i=1}^{n} a_{i} \tau_{k-i} \\
& +\sum_{j=1}^{m} b_{j}\left(\sum_{i=1}^{n} a_{i} \tau_{k-(i+j-1)}-\sum_{i=1}^{n} a_{i} \tau_{k-(i+j)}\right)
\end{aligned}
$$

\subsection{Practical Justification for the Reformulated Model}

In this subsection, we provide some practical justification for the new assumptions of our reformulated model. Actually we may state the following arguments:

1. The delayed model (or memory model) is quite interesting from the mathematical point of view. The higher order polynomials produce oscillatory trajectories for the solutions and consequently for the national income values.

2. The "delay" concept has also been suggested by previous papers. We refer to Chow (1985), who suggests the delayed information as a tool to explain and support the statistical data of Chinese economy for the years 1932-1982.

3. The national accounts of main countries are closing with substantial time delay after the calendar year end. So, the information used in next year's accounts are estimations or closed values from past years.

4. Delayed information may be used in financial projections of national accounts.

5. Consumption may depend not only on current year's experience but also on previous years. Customers remember the level of their income not only of the current year but also from previous years. So, they adjust their behavior accordingly.

6. Private investment may also consider the level of national economy of previous years. An investor keeps in mind not only the current level of economy but the time sequence and the corresponding trajectory of national economy all the recent years.

7. Governmental expenditure is not normally constant (as Samuelson's model suggests) but certainly is a variable item fully controlled from the government taking into account the past experience and also the future targets for the national economy.

8. The multiplier and accelerator factors are not constant over time but may fluctuate and most probably behave as random variables. 


\section{Stability Investigation}

The full stability investigation for the general case described by Eq. (6) is quite difficult and probably not so informative. Actually, the full investigation leads to higher order polynomials where there are no analytical solutions. Of course, numerical solutions and approximations may be employed for those cases. Here we restrict our attention to two special cases. Firstly, we consider the following case.

Consumption $C_{k}$ depends only on past year's income value while private investment $I_{k}$ depends on consumption changes within the last two years and governmental expenditure $G_{k}$ depends on past year's income value.

This means that for (5) we have $n=1, a_{1}=a, m=2, b_{1}=b_{2}=b, \bar{G}_{k}=0$, $p=1, c_{1}=c$. The national income is then determined via the following third-order linear difference equation:

$$
T_{k}=(a+c+a b) T_{k-1}-a b T_{k-3},
$$

or, equivalently,

$$
T_{k+3}-(a+c+a b) T_{k+2}+a b T_{k}=0 .
$$

By investigating the stability of (8) we arrive at Theorem 3.1. Firstly, we state the two following well-known and useful lemmas.

Lemma 3.1 (See Azzo and Houpis 1995; Dorf 1983; Kuo 1996; Ogata 1987) The homogeneous difference equation (8) is asymptotically stable, if and only if all roots of the equation

$$
s^{3}-(a+a b+c) s^{2}+a b=0,
$$

lie within the open disk,

$$
S=\{s \in \mathbb{C}:|s|<1\} .
$$

Lemma 3.2 (See Azzo and Houpis 1995; Dorf 1983; Kuo 1996; Ogata 1987) By means of the bilinear transformation

$$
s=\frac{w+1}{w-1},
$$

the open disc (9) is transformed into the open half plane

$$
W=\{w \in \mathbb{C}: \operatorname{Re}(w)<0\} .
$$

Theorem 3.1 Consider Eq. (8). Then for $0<a<1, b, c>0$, the equilibrium $T^{*}=0$ is asymptotically stable if and only if

$$
\begin{aligned}
a+c & <1 ; \\
a+c+4 a b & <3 ; \\
2 a^{2} b^{2}+a b c+a^{2} b & <1 .
\end{aligned}
$$


Proof By investigating the stability of (8), for $a+c \neq 1$ and the equilibrium $T^{*}$ of (8) we have

$$
T^{*}-(a+c+a b) T^{*}+a b T^{*}=0,
$$

or, equivalently,

$$
T^{*}=0 \text {. }
$$

The characteristic equation of (8) is

$$
s^{3}-(a+a b+c) s^{2}+a b=0 .
$$

By applying the transformation (10) into Eq. (8) we get the polynomial

$$
\begin{aligned}
& (1-a-c) w^{3}+(3-a-c-4 a b) w^{2} \\
& \quad+(3+a+c+4 a b) w+(1+a+c)=0 .
\end{aligned}
$$

A simple procedure to determine the existence of roots with negative real parts is needed according to Lemmas 3.1 and 3.2. Routh's criterion is a simple method of determining the number of these roots. The coefficients of $w$ 's and all powers from $w^{3}$ to $w^{0}$ must be present in the characteristic equation. A necessary but not sufficient condition for stable roots is that all coefficients in (11) must be positive. If any coefficients, other than $1+a+c$, are zero or if all the coefficients do not have the same sign, then there are pure imaginary roots or roots with positive real parts and the equation is not asymptotically stable. Thus

$$
\begin{array}{r}
a+c<1, \\
a+c+4 a b<3 .
\end{array}
$$

The coefficients of the characteristic equation are arranged in the pattern shown in the first rows of the following Routhian array. These coefficients are then used to evaluate the rest of the constants to complete the array.

$$
\begin{array}{c|cc}
w^{3} & A & C \\
w^{2} & B & D \\
w^{1} & E & 0 \\
w^{0} & F & 0
\end{array} \mid .
$$

The constants $A, B, C, D$ are defined as follows:

$$
\begin{aligned}
& A=1-a-c, \\
& B=3-a-c-4 a b, \\
& C=3+a+c+4 a b, \\
& D=1+a+c,
\end{aligned}
$$


and

$$
\begin{aligned}
& E=-\frac{\operatorname{det}\left(\begin{array}{ll}
A & C \\
B & D
\end{array}\right)}{B}=-\frac{A D-C B}{B}, \\
& F=-\frac{\operatorname{det}\left(\begin{array}{ll}
B & D \\
E & 0
\end{array}\right)}{E}=D .
\end{aligned}
$$

Routh's criterion states that the number of roots of the characteristic equation with positive real parts is equal to the number of changes of sign of the coefficients in the first column; see Azzo and Houpis (1995), Dorf (1983), Kuo (1996), Ogata (1987). Therefore, the difference equation is stable if all terms in the first column have the same sign. Since $A, B, F$ are positive, we have

$$
-\frac{A D-C B}{B}>0
$$

or, equivalently,

$$
2 a^{2} b^{2}+a b c+a^{2} b<1 .
$$

The proof is completed.

Next we will consider the following case.

Consumption $C_{k}$ depends on the last two year's income values while private investment $I_{k}$ depends on consumption changes within the last two years and governmental expenditure $G_{k}$ depends on past year's income value.

This means that for (5) we have $n=2, m=2, \bar{G}_{k}=0, p=1, c_{1}=c, a_{1}>a_{2}$, $b_{1}>b_{2}$. We have also to assume $a_{1}>a_{2}$ and $b_{1}>b_{2}$ because we normal weight heavier the most recent experience. The national income is determined via the following fourth-order linear difference equation:

$$
\begin{aligned}
T_{k}= & a_{1} T_{k-1}+a_{2} T_{k-2}+c T_{k-1}+b_{1}\left[a_{1} T_{k-1}+\left(a_{2}-a_{1}\right) T_{k-2}-a_{2} T_{k-3}\right] \\
& +b_{2}\left[a_{1} T_{k-2}+\left(a_{2}-a_{1}\right) T_{k-3}-a_{2} T_{k-4}\right],
\end{aligned}
$$

or, equivalently,

$$
\begin{aligned}
& T_{k+4}-\left(a_{1}+c+b_{1} a_{1}\right) T_{k+3}-\left[a_{2}+b_{1}\left(a_{2}-a_{1}\right)+b_{2} a_{1}\right] T_{k+2} \\
& \quad-\left[b_{2}\left(a_{2}-a_{1}\right)-b_{1} a_{2}\right] T_{k+1}+a_{2} b_{2} T_{k}=0 .
\end{aligned}
$$

By investigating the stability of the above equation we arrive at the following theorem.

Theorem 3.2 Consider Eq. (12). Then for $0<a_{2}<a_{1}<1,0<b_{2}<b_{1}<3$, and $c>0$, the equilibrium $T^{*}=0$ is asymptotically stable if and only if

$$
\begin{aligned}
& A>0 ; \\
& B>0 ;
\end{aligned}
$$




$$
\begin{gathered}
E>0 ; \\
B C>A D ; \\
D F>B E .
\end{gathered}
$$

Here the constants $A, B, C, D, E, F$, are defined as follows:

$$
\begin{aligned}
& A=1-c-a_{1}-a_{2}, \\
& B=4-2 c-2 a_{1}-2 a_{1} b_{1}-2 a_{2} b_{2}-2 a_{1} b_{2}-2 b_{1} a_{2}, \\
& C=6+2 a_{2}+2 b_{1} a_{2}-2 a_{1} b_{1}+2 a_{1} b_{2}+6 a_{2} b_{2}, \\
& D=4+2 c+2 a_{1}+2 a_{1} b_{1}-6 a_{2} b_{2}+2 a_{1} b_{2}+2 b_{1} a_{2}, \\
& E=1+c+a_{1}-a_{2}+2 b_{1} a_{1}-2 b_{1} a_{2}-2 b_{2} a_{1}+2 a_{2} b_{2}, \\
& F=-\frac{A D-B C}{B} .
\end{aligned}
$$

Proof By investigating the stability of (12), for $a_{1}+a_{2}+c \neq 1$ and the equilibrium $T^{*}$ of (12) we have

$$
\begin{aligned}
& T^{*}-\left(a_{1}+c+b_{1} a_{1}\right) T^{*}-\left[a_{2}+b_{1}\left(a_{2}-a_{1}\right)+b_{2} a_{1}\right] T^{*} \\
& \quad-\left[b_{2}\left(a_{2}-a_{1}\right)-b_{1} a_{2}\right] T^{*}+a_{2} b_{2} T^{*}=0,
\end{aligned}
$$

or, equivalently,

$$
T^{*}=0 \text {. }
$$

The characteristic equation of (12) is

$$
\begin{aligned}
s^{4}- & \left(a_{1}+c+b_{1} a_{1}\right) s^{3}-\left[a_{2}+b_{1}\left(a_{2}-a_{1}\right)+b_{2} a_{1}\right] s^{2} \\
- & {\left[b_{2}\left(a_{2}-a_{1}\right)-b_{1} a_{2}\right] s+a_{2} b_{2}=0 . }
\end{aligned}
$$

The homogeneous difference equation (12) is asymptotically stable if and only if all roots of Eq. (14) lie within the open disk, $|s|<1$. By means of the bilinear transformation $s=\frac{w+1}{w-1}$, the open disc is transformed into the open half plane $\operatorname{Re}(w)<0$. Hence by applying the transformation in (10) we get the polynomial

$$
A w^{4}+B w^{3}+C w^{2}+D w+E=0 .
$$

Here $A, B, C, D, E$ are defined in (13). Again we apply the Routh's criterion in order to determine the number of roots with negative real parts. It is fairly easy to show that $C>0$ and $D>0 . C=6+2 a_{2}+2 b_{1} a_{2}-2 a_{1} b_{1}+2 a_{1} b_{2}+6 a_{2} b_{2}>6-2 a_{1} b_{1}>$ $6-2 a_{1} b_{1}>6-2 b_{1}>0$ and $D=4+2 c+2 a_{1}+2 a_{1} b_{1}-6 a_{2} b_{2}+2 a_{1} b_{2}+2 b_{1} a_{2}>$ $6+2 a_{2}+2 a_{2} b_{2}-6 a_{2} b_{2}+2 a_{2} b_{2}+2 b_{2} a_{2}>6+2 a_{2}>0$. Since $C, D>0$, and a necessary but not sufficient condition for stable roots is that all coefficients in (15) 
must have the same sign, we require

$$
\begin{aligned}
& A>0, \\
& B>0, \\
& E>0 .
\end{aligned}
$$

The coefficients of the characteristic equation are arranged in the pattern shown in the first rows of the following Routhian array. These coefficients are then used to evaluate the rest of the constants to complete the array.

$$
\begin{array}{l|lll}
w^{4} & A & C & E \\
w^{3} & B & D & 0 \\
w^{2} & F & G & 0 \\
w^{1} & H & 0 & 0 \\
w^{0} & J & 0 & 0
\end{array} .
$$

The constant $F$ is defined in (13). The constants $G, H, J$ are defined as follows:

$$
\begin{aligned}
G & =-\frac{\operatorname{det}\left(\begin{array}{ll}
A & B \\
E & 0
\end{array}\right)}{B}=E, \\
H & =-\frac{\operatorname{det}\left(\begin{array}{ll}
B & D \\
F & G
\end{array}\right)}{F}=-\frac{B G-F D}{F}=-\frac{B E-F D}{F}, \\
J & =-\frac{\operatorname{det}\left(\begin{array}{ll}
F & G \\
H & 0
\end{array}\right)}{H}=G=E .
\end{aligned}
$$

From Routh's criterion equilibrium of the difference equation is stable if all terms in the first column have the same sign. While $A, B, E$ are positive,

$$
\begin{aligned}
& F>0, \\
& H>0,
\end{aligned}
$$

or, equivalently,

$$
\begin{aligned}
& B C>A D, \\
& D F>B E .
\end{aligned}
$$

The proof is completed.

Remark 3.1 There are available three stability tests. The first method that we used in the proofs of Theorems 3.1 and 3.2 is based on the bilinear transformation coupled with the Routh stability criterion. The other two are the Shur-Cohn stability test and the Jury stability test. The computations required in the Jury test are simpler than those required in the Shur-Cohn test but according to our view and as commented also in Ogata (1987), the first method is simpler and more straightforward.

Remark 3.2 In Sect. 5 we provide indicative examples for the stability conditions derived in Theorems 3.1 and 3.2. 


\section{Controllability and State Feedback}

In this section we introduce concepts and results of linear control theory for time invariant linear discrete state equations. We investigate two other special cases in which the $c_{i}$ parameters are all zero. So, the governmental expenditure depends only on $\bar{G}_{k}$ which is a fully controlled variable as also mentioned in Sect. 2 when formulating our model. Firstly, we consider the following case.

Consumption $C_{k}$ depends on past year's income value while private investment $I_{k}$ depends on consumption changes within the last two years and governmental expenditure $G_{k}$ does not depend on any past values.

This means that for (5) we have $n=1, a_{1}=a, m=2, b_{1}=b_{2}=b, c_{1}=c_{2}=$ $\cdots=c_{p}=0$. The national income is then determined via the following third-order linear difference equation:

$$
T_{k+3}-(a+a b) T_{k+2}+a b T_{k}=\bar{G}_{k} .
$$

By adopting the following notation:

$$
\begin{aligned}
T_{k} & =y_{k}^{1}, \\
T_{k+1} & =y_{k}^{2}, \\
T_{k+2} & =y_{k}^{3},
\end{aligned}
$$

and

$$
\begin{aligned}
& T_{k+1}=y_{k+1}^{1}=y_{k}^{2}, \\
& T_{k+2}=y_{k+1}^{2}=y_{k}^{3}, \\
& T_{k+3}=y_{k+1}^{3}=a(1+b) y_{k}^{3}-a b y_{k}^{1}+\bar{G}_{k},
\end{aligned}
$$

Eq. (16) takes the form

$$
Y_{k+1}=P Y_{k}+Q \bar{G}_{k},
$$

where

$$
Y_{k}=\left[\begin{array}{c}
y_{k}^{1} \\
y_{k}^{2} \\
y_{k}^{3}
\end{array}\right], \quad P=\left[\begin{array}{ccc}
0 & 1 & 0 \\
0 & 0 & 1 \\
-a b & 0 & a b+a
\end{array}\right], \quad Q=\left[\begin{array}{l}
0 \\
0 \\
1
\end{array}\right] .
$$

This is a linear discrete time control system with input vector $\bar{G}_{k}$. Linear control involves modification of the behavior of a given system by applying state feedback. The state feedback replaces the input $\bar{G}_{k}$ by

$$
\bar{G}_{k}=-K Y_{k},
$$


where

$$
K=\left[\begin{array}{lll}
K_{1} & K_{2} & K_{3}
\end{array}\right]
$$

and the system takes the form

$$
Y_{k+1}=(P-Q K) Y_{k}
$$

The basic problem is that of choosing a state feedback $K$ such that the resulting (closed loop equation) is stable. The stabilization in the time invariant case is via results on eigenvalue placement in the complex plane. In our situation eigenvalues of the closed loop system are specified to have modulus less than unity for stability. We can state the following theorem.

Theorem 4.1 Assume the system (17). Then there exists a state feedback law in the form of

$$
\bar{G}_{k}=-K Y_{k},
$$

where

$$
K=\left[\begin{array}{lll}
-\mu_{1} \mu_{2} \mu_{3}-a b & \mu_{1} \mu_{2}+\mu_{1} \mu_{3}+\mu_{2} \mu_{3} & a+a b-\left(\mu_{1}+\mu_{2}+\mu_{3}\right)
\end{array}\right],
$$

such that the eigenvalues of the closed loop system can be assigned arbitrarily as $\mu_{1}$, $\mu_{2}, \mu_{3}$.

Proof It is well known that for a system in the form of (17) there exists a state feedback law if and only if the system is completely controllable. The necessary and sufficient condition for complete controllability is

$$
\operatorname{rank}\left[\begin{array}{lll}
Q & P Q & P^{2} Q
\end{array}\right]=3 .
$$

Since

$$
\operatorname{det}\left[\begin{array}{lll}
Q & P Q & P^{2} Q
\end{array}\right]=\operatorname{det}\left[\begin{array}{ccc}
0 & 0 & 1 \\
0 & 1 & a+a b \\
1 & a b+a & (a b+a)^{2}
\end{array}\right]=-1 \neq 0
$$

the system (17) is controllable with state feedback of the form

$$
\bar{G}_{k}=-K Y_{k},
$$

where

$$
K=\left[\begin{array}{lll}
K_{1} & K_{2} & K_{3}
\end{array}\right] .
$$

Then the closed loop system is of the form

$$
Y_{k+1}=(P-Q K) Y_{k} .
$$


And thus we have

$$
P-Q K=\left[\begin{array}{ccc}
0 & 1 & 0 \\
0 & 0 & 1 \\
-a b & 0 & a+a b
\end{array}\right]-\left[\begin{array}{l}
0 \\
0 \\
1
\end{array}\right]\left[\begin{array}{lll}
K_{1} & K_{2} & K_{3}
\end{array}\right],
$$

or, equivalently,

$$
P-Q K=\left[\begin{array}{ccc}
0 & 1 & 0 \\
0 & 0 & 1 \\
-a b-K_{1} & -K_{2} & a+a b-K_{3}
\end{array}\right]
$$

with characteristic equation

$$
\Pi(z)=\operatorname{det}\left(z I_{3}-P+Q K\right)=z^{3}+\left(K_{3}-a-a b\right) z^{2}+K_{2} z+a b+K_{1} .
$$

If we have $\mu_{1}, \mu_{2}$, and $\mu_{3}$ as eigenvalues of the closed loop system, then

$$
\begin{aligned}
\Phi(z) & =\left(z-\mu_{1}\right)\left(z-\mu_{2}\right)\left(z-\mu_{3}\right) \\
& =z^{3}-\left(\mu_{1}+\mu_{2}+\mu_{3}\right) z^{2}+\left(\mu_{1} \mu_{2}+\mu_{1} \mu_{3}+\mu_{2} \mu_{3}\right) z-\mu_{1} \mu_{2} \mu_{3} .
\end{aligned}
$$

We require

$$
\Pi(z) \equiv \Phi(z)
$$

or, equivalently,

$$
\begin{aligned}
-a b-a+K_{3} & =-\left(\mu_{1}+\mu_{2}+\mu_{3}\right), \\
K_{2} & =\mu_{1} \mu_{2}+\mu_{1} \mu_{3}+\mu_{2} \mu_{3}, \\
a b+K_{1} & =-\mu_{1} \mu_{2} \mu_{3}
\end{aligned}
$$

and thus the matrix $K$ takes the values

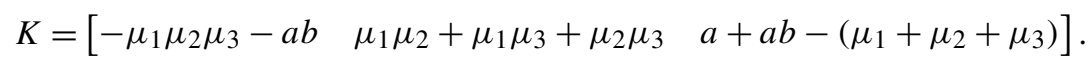

The proof is completed.

So returning to the difference equation (16), $\bar{G}_{k}$ is determined via the relation (18), or, equivalently,

$$
\begin{aligned}
\bar{G}_{k}= & -\left(-\mu_{1} \mu_{2} \mu_{3}-a b\right) T_{k}-\left(\mu_{1} \mu_{2}+\mu_{1} \mu_{3}+\mu_{2} \mu_{3}\right) T_{k+1} \\
& -\left(a+a b-\left(\mu_{1}+\mu_{2}+\mu_{3}\right)\right) T_{k+2}
\end{aligned}
$$

and by replacing it into Eq. (16) we get

$$
T_{k+3}-\left(\mu_{1}+\mu_{2}+\mu_{3}\right) T_{k+2}+\left(\mu_{1} \mu_{2}+\mu_{1} \mu_{3}+\mu_{2} \mu_{3}\right) T_{k+1}-\mu_{1} \mu_{2} \mu_{3} T_{k}=0 .
$$


Example 4.1 We assume the difference equation (16). If for the system (17) we require the eigenvalue $\mu_{1}=\mu_{2}=0$ with algebraic multiplicity 2 and $\mu_{3}=a$ then

$$
K=\left[\begin{array}{lll}
-a b & 0 & a b
\end{array}\right]
$$

and

$$
\bar{G}_{k}=\left[\begin{array}{lll}
a b & 0 & -a b
\end{array}\right] Y_{k},
$$

or, equivalently,

$$
\bar{G}_{k}=-a b T_{k}-a b T_{k+2} .
$$

By replacing the above expression into the difference equation (19) we arrive at

$$
T_{k+3}-a T_{k+2}=0 .
$$

The equilibrium of the above difference equation is asymptotically stable since the roots of its characteristic polynomial are $\mu_{1}=\mu_{2}=0$ and $\mu_{3}=a$. The example is completed.

Next we will consider the following case.

We assume that consumption $C_{k}$ depends on the last two year's income values, while private investment $I_{k}$ depends on consumption changes within the last two years and governmental expenditure does not depend on past years value, i.e. it depends only on $\bar{G}_{k}$.

This means that for Eq. (5) we have $n=2, m=2, c_{1}=c_{2}=\cdots=c_{p}=0$. The national income in this case is determined via the following fourth-order linear difference equation:

$$
\begin{aligned}
& T_{k+4}-\left(a_{1}+b_{1} a_{1}\right) T_{k+3}-\left[a_{2}+b_{1}\left(a_{2}-a_{1}\right)+b_{2} a_{1}\right] T_{k+2} \\
& \quad-\left[b_{2}\left(a_{2}-a_{1}\right)-b_{1} a_{2}\right] T_{k+1}+a_{2} b_{2} T_{k}=\bar{G}_{k} .
\end{aligned}
$$

We adopt the following notation:

$$
\begin{aligned}
T_{k} & =y_{k}^{1}, \\
T_{k+1} & =y_{k}^{2}, \\
T_{k+2} & =y_{k}^{3}, \\
T_{k+3} & =y_{k}^{4},
\end{aligned}
$$

and

$$
\begin{aligned}
& T_{k+1}=y_{k+1}^{1}=y_{k}^{2}, \\
& T_{k+2}=y_{k+1}^{2}=y_{k}^{3},
\end{aligned}
$$




$$
\begin{aligned}
& T_{k+3}=y_{k+1}^{3}=y_{k}^{4}, \\
& T_{k+4}=y_{k+1}^{4},
\end{aligned}
$$

or, in matrix form,

$$
Y_{k+1}=P Y_{k}+Q \bar{G}_{k}
$$

where

$$
\begin{aligned}
& Y_{k}=\left[\begin{array}{c}
y_{k}^{1} \\
y_{k}^{2} \\
y_{k}^{3} \\
y_{k}^{4}
\end{array}\right] \\
& P=\left[\begin{array}{cccc}
0 & 1 & 0 & 0 \\
0 & 0 & 1 & 0 \\
0 & 0 & 0 & 1 \\
-a_{2} b_{2} & b_{2}\left(a_{2}-a_{1}\right)-b_{1} a_{2} & a_{2}+b_{1}\left(a_{2}-a_{1}\right)+b_{2} a_{1} & a_{1}\left(1+b_{1}\right)
\end{array}\right] \text {, } \\
& Q=\left[\begin{array}{l}
0 \\
0 \\
0 \\
1
\end{array}\right] \text {. }
\end{aligned}
$$

We can state the following theorem.

Theorem 4.2 Assume the system (21). Then there exists a state feedback law of the form

$$
\bar{G}_{k}=-K Y_{k}
$$

where

$$
K=\left[\begin{array}{c}
\lambda_{1} \lambda_{2} \lambda_{3} \lambda_{4}-a_{2} b_{2} \\
b_{2}\left(a_{2}-a_{1}\right)-b_{1} a_{2}-\left(\lambda_{1} \lambda_{2} \lambda_{3}+\lambda_{1} \lambda_{2} \lambda_{4}+\lambda_{1} \lambda_{3} \lambda_{4}+\lambda_{2} \lambda_{3} \lambda_{4}\right) \\
-\left[a_{2}+b_{1}\left(a_{2}-a_{1}\right)+b_{2} a_{1}\right]-\left(\lambda_{1} \lambda_{2}+\lambda_{1} \lambda_{3}+\lambda_{1} \lambda_{4}+\lambda_{2} \lambda_{3}+\lambda_{2} \lambda_{4}+\lambda_{3} \lambda_{4}\right) \\
a_{1}\left(1+b_{1}\right)-\left(\lambda_{1}+\lambda_{2}+\lambda_{3}+\lambda_{4}\right)
\end{array}\right]^{T},
$$

such that the eigenvalues of the closed loop system can be assigned arbitrarily as $\lambda_{1}$, $\lambda_{2}, \lambda_{3}$, and $\lambda_{4}$.

Proof The necessary and sufficient condition for system (21) to have complete controllability is

$$
\operatorname{rank}\left[\begin{array}{llll}
Q & P Q & P^{2} Q & P^{3} Q
\end{array}\right]=4
$$

Since

$$
\operatorname{det}\left[Q \quad P Q \quad P^{2} Q \quad P^{3} Q\right]=1 \neq 0,
$$

the system (21) is controllable and by replacing

$$
\bar{G}_{k}=-K Y_{k},
$$


where

$$
K=\left[\begin{array}{llll}
K_{1} & K_{2} & K_{3} & K_{4}
\end{array}\right],
$$

the system takes the form

$$
Y_{k+1}=(P-Q K) Y_{k} .
$$

Thus we have

$$
\begin{aligned}
P & =Q K \\
= & {\left[\begin{array}{cccc}
0 & 1 & 0 & 0 \\
0 & 0 & 1 & 0 \\
0 & 0 & 0 & 1 \\
-a_{2} b_{2} & b_{2}\left(a_{2}-a_{1}\right)-b_{1} a_{2} & a_{2}+b_{1}\left(a_{2}-a_{1}\right)+b_{2} a_{1} & a_{1}\left(1+b_{1}\right)
\end{array}\right] } \\
& -\left[\begin{array}{l}
0 \\
0 \\
0 \\
1
\end{array}\right]\left[\begin{array}{llll}
K_{1} & K_{2} & K_{3} & K_{4}
\end{array}\right],
\end{aligned}
$$

or, equivalently,

$$
\begin{aligned}
& P-Q K \\
& =\left[\begin{array}{ccc}
0 & 1 \\
0 & 0 & \\
0 & 0 & 0 \\
-a_{2} b_{2}-K_{1} & b_{2}\left(a_{2}-a_{1}\right)-b_{1} a_{2}-K_{2} & \\
0 & 0 \\
1 & 1 \\
0 & a_{2}+b_{1}\left(a_{2}-a_{1}\right)+b_{2} a_{1}-K_{3} & a_{1}\left(1+b_{1}\right)-K_{4}
\end{array}\right] .
\end{aligned}
$$

Let $\lambda_{1}, \lambda_{2}, \lambda_{3}$, and $\lambda_{4}$ be the eigenvalues of the matrix $P-Q K$ with modulus less than 1 and let there also be roots of the polynomial

$$
\begin{gathered}
z^{4}-\left(\lambda_{1}+\lambda_{2}+\lambda_{3}+\lambda_{4}\right) z^{3}+\left(\lambda_{1} \lambda_{2}+\lambda_{1} \lambda_{3}+\lambda_{1} \lambda_{4}+\lambda_{2} \lambda_{3}+\lambda_{2} \lambda_{4}+\lambda_{3} \lambda_{4}\right) z^{2} \\
-\left(\lambda_{1} \lambda_{2} \lambda_{3}+\lambda_{1} \lambda_{2} \lambda_{4}+\lambda_{1} \lambda_{3} \lambda_{4}+\lambda_{2} \lambda_{3} \lambda_{4}\right) z+\lambda_{1} \lambda_{2} \lambda_{3} \lambda_{4}=0 .
\end{gathered}
$$

The characteristic polynomial of the matrix $P-Q K$ is

$$
\operatorname{det}\left(z I_{4}-P+Q K\right)=0
$$

or, equivalently,

$$
\begin{aligned}
z^{4}- & \left(a_{1}\left(1+b_{1}\right)-K_{4}\right) z^{3}-\left[a_{2}+b_{1}\left(a_{2}-a_{1}\right)+b_{2} a_{1}-K_{3}\right] z^{2} \\
- & {\left[b_{2}\left(a_{2}-a_{1}\right)-b_{1} a_{2}-K_{2}\right] z+a_{2} b_{2}+K_{1}=0, }
\end{aligned}
$$


and thus the matrix $K$ takes the values

$$
K=\left[\begin{array}{c}
\lambda_{1} \lambda_{2} \lambda_{3} \lambda_{4}-a_{2} b_{2} \\
b_{2}\left(a_{2}-a_{1}\right)-b_{1} a_{2}-\left(\lambda_{1} \lambda_{2} \lambda_{3}+\lambda_{1} \lambda_{2} \lambda_{4}+\lambda_{1} \lambda_{3} \lambda_{4}+\lambda_{2} \lambda_{3} \lambda_{4}\right) \\
-\left[a_{2}+b_{1}\left(a_{2}-a_{1}\right)+b_{2} a_{1}\right]-\left(\lambda_{1} \lambda_{2}+\lambda_{1} \lambda_{3}+\lambda_{1} \lambda_{4}+\lambda_{2} \lambda_{3}+\lambda_{2} \lambda_{4}+\lambda_{3} \lambda_{4}\right) \\
a_{1}\left(1+b_{1}\right)-\left(\lambda_{1}+\lambda_{2}+\lambda_{3}+\lambda_{4}\right)
\end{array}\right]^{T} .
$$

The proof is completed.

So, returning to the difference equation $(20), \bar{G}_{k}$ is determined via the relation (22), or, equivalently,

$$
\begin{aligned}
\bar{G}_{k}= & -\left(\lambda_{1} \lambda_{2} \lambda_{3} \lambda_{4}-a_{2} b_{2}\right) T_{k} \\
& -\left[b_{2}\left(a_{2}-a_{1}\right)-b_{1} a_{2}-\left(\lambda_{1} \lambda_{2} \lambda_{3}+\lambda_{1} \lambda_{2} \lambda_{4}+\lambda_{1} \lambda_{3} \lambda_{4}+\lambda_{2} \lambda_{3} \lambda_{4}\right)\right] T_{k+1} \\
& -\left[-\left(a_{2}+b_{1}\left(a_{2}-a_{1}\right)+b_{2} a_{1}\right)\right. \\
& \left.-\left(\lambda_{1} \lambda_{2}+\lambda_{1} \lambda_{3}+\lambda_{1} \lambda_{4}+\lambda_{2} \lambda_{3}+\lambda_{2} \lambda_{4}+\lambda_{3} \lambda_{4}\right)\right] T_{k+2} \\
& -\left(a_{1}\left(1+b_{1}\right)-\left(\lambda_{1}+\lambda_{2}+\lambda_{3}+\lambda_{4}\right)\right) T_{k+3}
\end{aligned}
$$

and by replacing the above expression into Eq. (20) we get

$$
\begin{aligned}
& T_{k+4}-\left(\lambda_{1}+\lambda_{2}+\lambda_{3}+\lambda_{4}\right) T_{k+3} \\
& \quad+\left(\lambda_{1} \lambda_{2}+\lambda_{1} \lambda_{3}+\lambda_{1} \lambda_{4}+\lambda_{2} \lambda_{3}+\lambda_{2} \lambda_{4}+\lambda_{3} \lambda_{4}\right) T_{k+2} \\
& \quad-\left(\lambda_{1} \lambda_{2} \lambda_{3}+\lambda_{1} \lambda_{2} \lambda_{4}+\lambda_{1} \lambda_{3} \lambda_{4}+\lambda_{2} \lambda_{3} \lambda_{4}\right) T_{k+1}+\left(\lambda_{1} \lambda_{2} \lambda_{3} \lambda_{4}\right) T_{k}=0 .
\end{aligned}
$$

Example 4.2 We assume the difference equation (20). If we require for system (21) its eigenvalues to be $\lambda_{1}=\lambda_{2}=\lambda_{3}=0$ with algebraic multiplicity 3 and $\lambda_{4}=a_{1}$ then

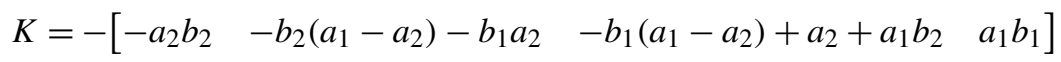

and

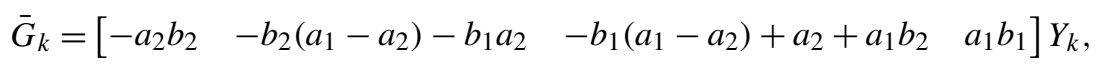

or, equivalently,

$$
\begin{aligned}
\bar{G}_{k}= & -a_{2} b_{2} T_{k}-\left(b_{2}\left(a_{1}-a_{2}\right)+b_{1} a_{2}\right) T_{k+1} \\
& +\left(-b_{1}\left(a_{1}-a_{2}\right)+a_{2}+a_{1} b_{2}\right) T_{k+2}+a_{1} b_{1} T_{k+3} .
\end{aligned}
$$

By replacing (23) into the difference equation (20) we arrive at

$$
T_{k+4}-a_{1} T_{k+3}=0 .
$$

The equilibrium of the above difference equation is asymptotically stable since the roots of the characteristic polynomial are $\lambda_{1}=\lambda_{2}=\lambda_{3}=0$ and $\lambda_{4}=a_{1}$. The example is completed. 


\section{Numerical Examples: System Design and Practical Implications}

In this section we present numerical examples in order to provide further insight and better understanding regarding the control actions, system design, and produced business cycles.

Example 5.1 Firstly, we will consider the first case described in Sect. 3 via the difference equation (8). We will provide an indicative numerical example by using typical values, refer to Chow (1985), for the basic parameters $a$ and $b$ of the model. Actually we assume that the expectations of multiplier and accelerator factors are the following:

$$
\mathbb{E}[A]=a=0.5, \quad \mathbb{E}[B]=b=0.3 .
$$

Then Eq. (8) takes the form

$$
T_{k+3}-(0.65+c) T_{k+2}+0.15 T_{k}=0 .
$$

We may use Theorem 3.1 and determine the value of parameter $c$ such that the system is stable. Note that $G_{k}=c T_{k-1}$ and thus through $c$ the government controls the relevant governmental expenditure $G_{k}$ in order to obtain asymptotic stability in the system of national economy. According to the conditions of Theorem 3.1 and the given values for $a$ and $b$, we derive the boundary values for parameter $c$. By substituting $a$ and $b$ into the conditions of Theorem 3.1 we get $c<0.5, c<1.9, c<5.8$, or, equivalently,

$$
c<0.5 \text {. }
$$

Obviously for practical reasons $c$ cannot be negative. The option of a zero $c$ parameter may also be considered. Actually, that means there is no governmental intervention into the economic system. Excluding this limited case, we obtain the following inequality condition for asymptotic stability:

$$
0<c<0.5 \text {. }
$$

So, as far as the annual governmental expenditure does not exceed the half of last year's national income value, the system remains stable. If the government tries to boost the economy injecting a high level (greater than $0.5 T_{k-1}$ ) of expenditure then the system becomes unstable.

Now, we can further design the value for parameter $c$ targeting to a high speed response system. That means that we are going to search which values of the $c$ parameter guarantee not only the stability of the system (i.e. the solution trajectories of the system converging to asymptotic stability points) but additionally ensures that solution trajectories return very quickly to the desired stability level.

The speed of the system's response is basically characterized by the maximum value $r$ of the following set:

$$
r=\max \left\{\left|r_{1}\right|,\left|r_{2}\right|,\left|r_{3}\right|\right\},
$$




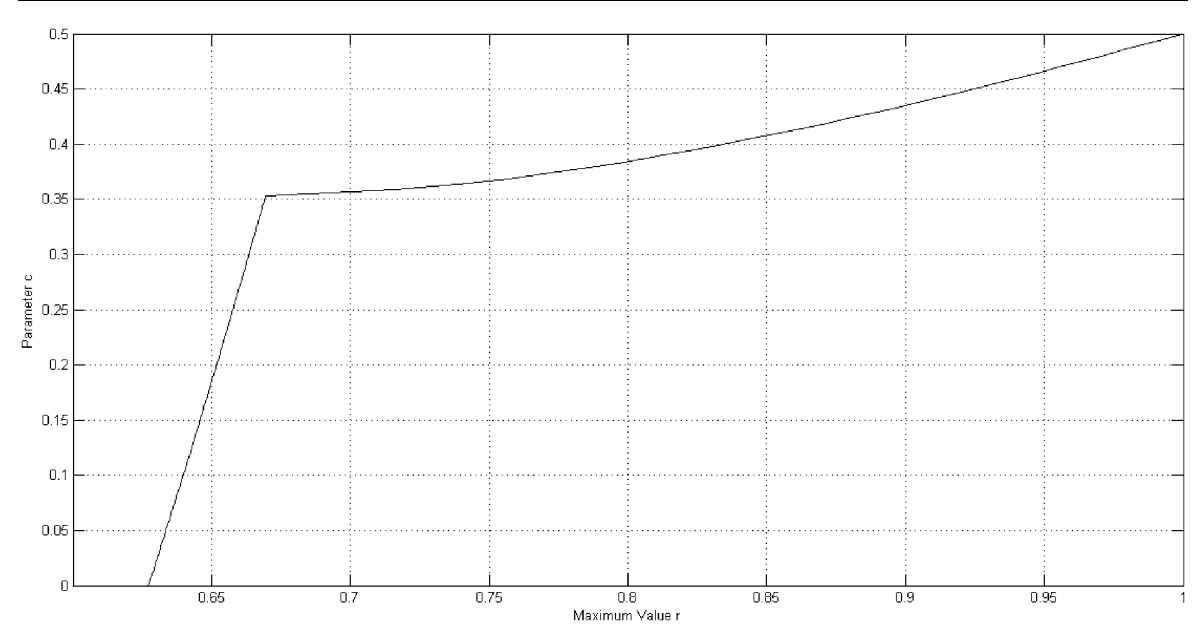

Fig. 1 Maximum value $r$, parameter $c$

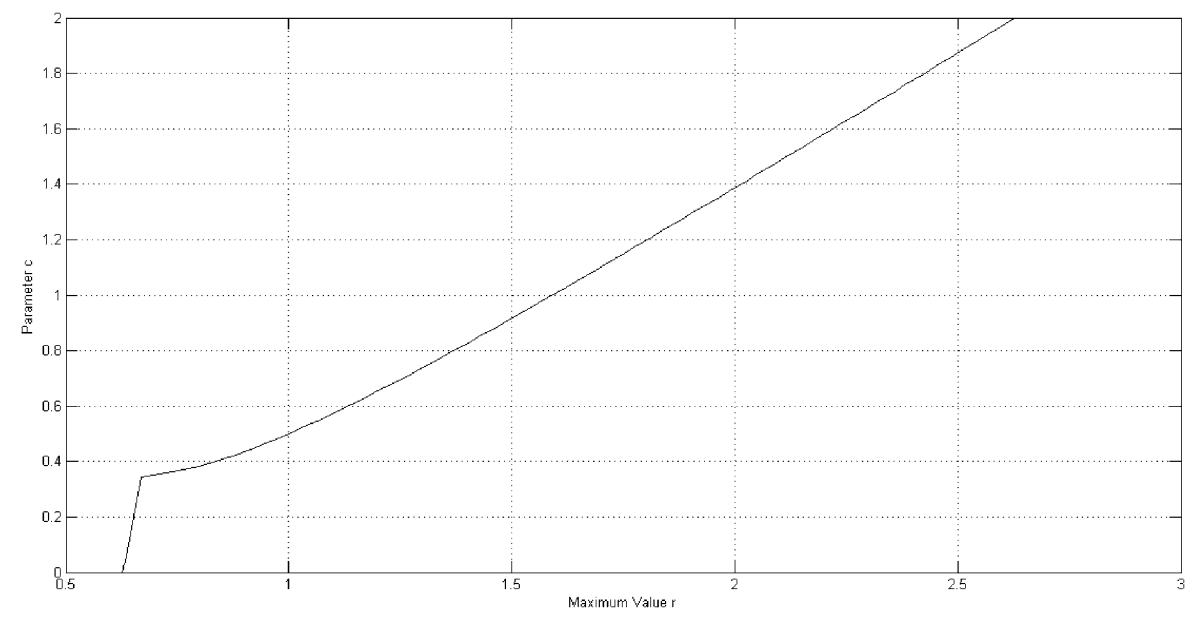

Fig. 2 Maximum value $r$, parameter $c$

where $r_{1}, r_{2}$, and $r_{3}$ are the roots of the respective characteristic polynomial

$$
s^{3}-(0.65+c) s^{2}+0.15=0
$$

associated with Eq. (8). So, high speed response coincides with minimizing the value of $r$; see Figs. 1, 2.

Table 1 shows some values of $r$ for some given values of the parameter $c$. As we observe from Figs. 1 and 2, there is an increasing linear pattern for the value of $r$ (see the following graph) exhibiting two different slopes in the intervals $(0.00,0.35)$ and $(0.35,0.50)$. Hence if we choose for example $c=0.35$, we guarantee asymptotic stability and also ensure that solution trajectories will converge fast to the equilibrium. 
Table 1 Parameter $c$, maximum value $r$

\begin{tabular}{ll}
\hline Parameter $c$ & Maximum value $r$ \\
\hline 0.05 & 0.6201 \\
0.1 & 0.6313 \\
0.15 & 0.6347 \\
0.2 & 0.6506 \\
0.25 & 0.6512 \\
0.3 & 0.6539 \\
0.35 & 0.6634 \\
0.4 & 0.8300 \\
0.45 & 0.9211 \\
0.5 & 1 \\
\hline
\end{tabular}

Indeed, Eq. (8) will then take the form

$$
T_{k+3}-T_{k+2}+0.15 T_{k}=0
$$

with respective solutions

$$
T_{k}=g_{1}(-0.335)^{k}+g_{2}(0.668-i 0.043)^{k}+g_{3}(0.668+i 0.043)^{k},
$$

where $g_{1}, g_{2}$, and $g_{3}$ are constants determined by the initial conditions.

Example 5.2 We will now consider the second special case as described in Sect. 3 via the difference equation (12). In line with the initial estimation for the basic values $a$ and $b$ in the previous example, now we assume that the values for the parameters $a_{1}, a_{2}, b_{1}$, and $b_{2}$ are

$$
\begin{aligned}
& \mathbb{E}\left[A_{1}\right]=a_{1}=0.35, \quad \mathbb{E}\left[A_{2}\right]=a_{2}=0.15, \\
& \mathbb{E}\left[B_{1}\right]=b_{1}=0.2, \quad \mathbb{E}\left[B_{2}\right]=b_{2}=0.1 .
\end{aligned}
$$

Note that $a_{1}+a_{2}=0.5=a$ and $b_{1}+b_{2}=0.3=b$. Again the governmental authorities have to control the parameter $c$ because $G_{k}=c T_{k-1}$.

By using Theorem 3.2 we observe that there is no viable solution for the system, i.e. we cannot determine a value for the parameter $c$ in order to obtain a stable model although the summation of $a_{1}$ and $a_{2}$ equals $a$, while the summation of $b_{1}$ and $b_{2}$ equals $b$. This means that the government should be very careful when deciding the intervention into the economy via the governmental expenditure and should investigate how much delayed information is enclosed in the current status of economy. Furthermore, it should be clarified how much of the volume of consumption of private investments may attributable to one, two or three years before and then decide the intervention into the system.

We assume now different values for the parameters $a_{1}, a_{2}, b_{1}$, and $b_{2}$

$$
\begin{array}{ll}
\mathbb{E}\left[A_{1}\right]=a_{1}=0.1, & \mathbb{E}\left[A_{2}\right]=a_{2}=0.4, \\
\mathbb{E}\left[B_{1}\right]=b_{1}=0.1, & \mathbb{E}\left[B_{2}\right]=b_{2}=0.2 .
\end{array}
$$


Note that $a_{1}+a_{2}=0.5=a$ and $b_{1}+b_{2}=0.3=b$. By replacing the above given values into Eq. (12) we get

$$
T_{k+4}-(0.11+c) T_{k+3}-0.45 T_{k+2}-0.02 T_{k+1}+0.08 T_{k}=0 .
$$

The constants $A, B, C, D, E, F$ that appear in Theorem 3.2 take the form

$$
\begin{aligned}
& A=0.5-c, \\
& B=3.5-2 c, \\
& C=6.66, \\
& D=3.86+2 c, \\
& E=c+0.76, \\
& F=-\frac{(0.5-c)(3.86+2 c)-(3.5-2 c) 6.66}{3.5-2 c} .
\end{aligned}
$$

By replacing the above values into the conditions of Theorem 3.2 and by taking into consideration that $c>0$, we get

$$
0<c<0.5
$$

We can further design the value for parameter $c$ targeting to a high speed response system. The speed of the system's response is basically characterized by the maximum value $r$ of the following set:

$$
r=\max \left\{\left|r_{1}\right|,\left|r_{2}\right|,\left|r_{3}\right|,\left|r_{4}\right|\right\},
$$

where $r_{1}, r_{2}, r_{3}$, and $r_{4}$ are the roots of the respective characteristic polynomial

$$
s^{4}-(0.11+c) s^{3}-0.45 s^{2}-0.02 s+0.08=0,
$$

associated with Eq. (12). So, high speed response coincides with minimizing the value of $r$; see Figs. 3, 4 .

Table 2 shows some values of $r$ for some given values of the parameter $c$. As we observe there is an increasing linear pattern for the value of $r$, see Figs. 3 and 4, exhibiting two different slopes in the intervals $(0.00,0.05)$ and $(0.05,0.50)$. Hence, considering the table and the Figs. 3 and 4, we may choose for example $c=0.05$. This value guarantees the asymptotic stability and also ensures that solution trajectories will converge fast to stability point. Equation (12) will then take the form

$$
T_{k+4}-0.16 T_{k+3}-0.45 T_{k+2}-0.02 T_{k}+0.08=0,
$$

with respective solutions

$$
T_{k}=g_{1}(0.5)^{k}+g_{2}(0.591)^{k}+g_{3}(-0.466-i 0.232)^{k}+g_{4}(-0.466+i 0.232)^{k} .
$$

Here $g_{1}, g_{2}, g_{3}$, and $g_{4}$ are constants determined by the initial conditions. 


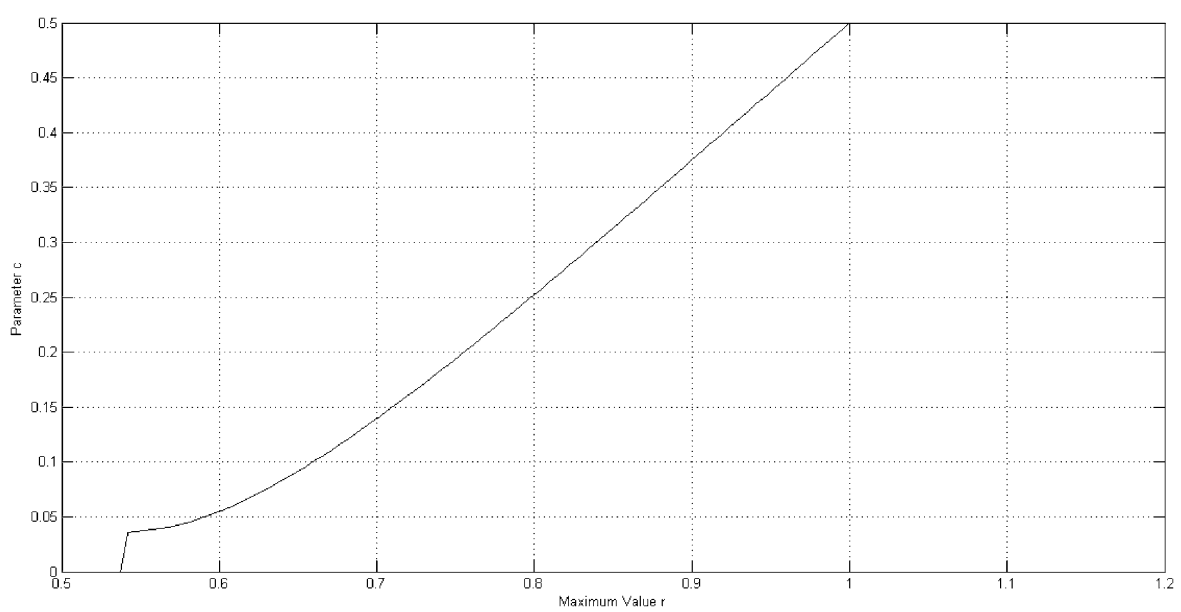

Fig. 3 Maximum value $r$, parameter $c$

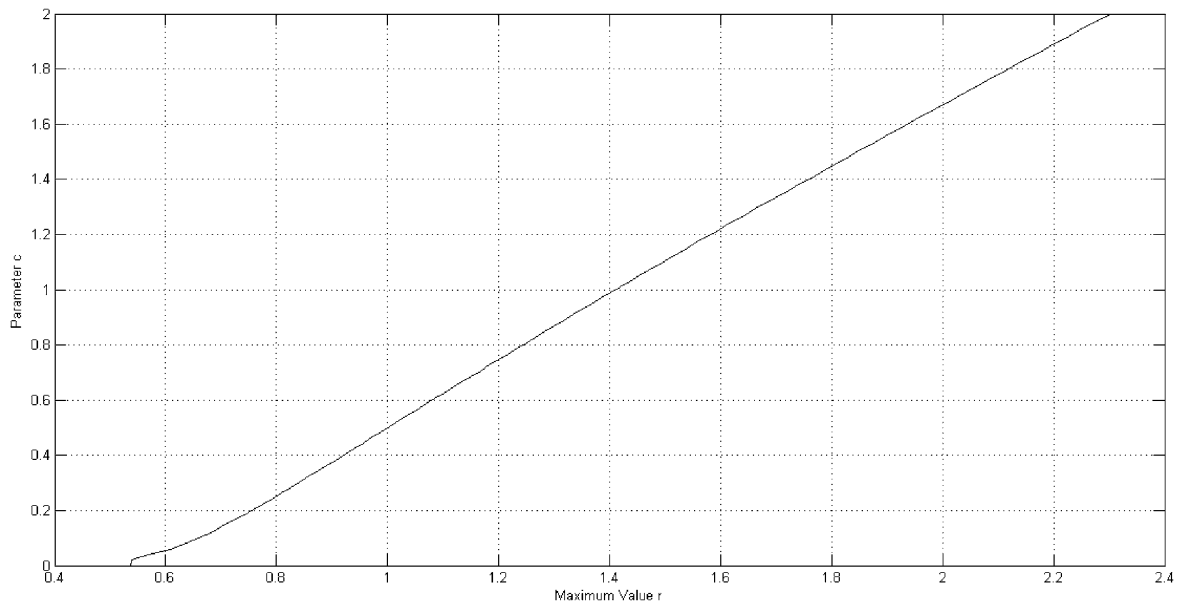

Fig. 4 Maximum value $r$, parameter $c$

\section{Conclusions}

Closing the paper, we may argue that the specific research effort is not only a theoretical extension of the basic version of Samuelson's model, but also a practical guide for controlling the parameters of national economy system. It investigates the stability and controllability concepts producing analytical conditions and solutions. These conditions enable the decision makers to adjust governmental expenditure such that to obtain stable trajectories for the expectation of national income values.

As we observe in Sect. 3, the government should have a reduced role and intervention into the system (given the specific values for $a, b$ the expectation of the multiplier and accelerator factor). Actually, the fastest stability response is obtained when there is a small governmental expenditure. Interpreting this result in economic 
Table 2 Parameter $c$, maximum value $r$

\begin{tabular}{ll}
\hline Parameter $c$ & Maximum value $r$ \\
\hline 0.05 & 0.591 \\
0.1 & 0.6509 \\
0.15 & 0.71 \\
0.2 & 0.7506 \\
0.25 & 0.7982 \\
0.3 & 0.8391 \\
0.35 & 0.88 \\
0.4 & 0.92 \\
0.45 & 0.96 \\
0.5 & 1 \\
\hline
\end{tabular}

terms we state the following case. Let us consider a national economy (as described in Sect. 3). The economy operates properly when a sudden change (input) arrives: e.g. a catastrophic event causes a substantial reduction into the system just for one year. The government aims to restore the initial level of economy. Someone may argue the "obvious-easy action" that government should proceed with a high level governmental expenditure next year in order to restore the economy. The results in Sect. 3 support the opposite. As stated there, the parameter $c$, of the governmental expenditure should not exceed the value 0.5 ; otherwise the economy will become unstable. That is, if the government spends a lot of money next year then this may guide the whole economy to instability.

Section 4 reveals the relationship between the controllability concept, feedback action and the way that we can design the trajectories of the economy. As we observe there, we may design the eigenvalues and consequently the solution trajectories. Considering the case study mentioned in the paragraph above, we may state that Sect. 4 may help the government not only to restore the stability into the economic system but also design the time path and the pattern of return.

Finally, a straightforward result is the evidence support for the fact that individuals and institutions have long "memory" and base their decisions for investment and/or consumption accordingly. The paper restores the inadequacy of the original Samuelson model that could not produce stable business cycles for the typical realistic values of the multiplier and accelerator parameters. That is obtained via the existence of higher order difference equations $(n \geq 3)$, resulting from the delay concept. The longer the delay, the higher order for the basic difference equation is obtained for the system. These higher order equations produce complex solutions and consequently oscillatory trajectories where under certain circumstances can be designed to be stable. Hence, the traditional model has two degrees of freedom (as the associated equation is a quadratic polynomial) exhibiting difficulties to design a stable trajectory path while the new version of the reformulated model has $n \geq 3$ degrees of freedom (as the associated equation is an $n$th order polynomial). 


\section{Competing Interests}

The authors declare that they have no competing interests.

\section{Authors' Contributions}

All authors contributed equally to the writing of this paper. All authors read and approved the final manuscript.

Acknowledgements The authors would like to express their sincere gratitude to the anonymous referees that clearly improved this article with their valuable suggestions. I. Dassios is supported by Science Foundation Ireland (award 09/SRC/E1780).

\section{References}

Azzo JD, Houpis CH (1995) Linear control system analysis and design, 4th edn. McGraw-Hill, New York Chari VV (1994) Optimal fiscal policy in a business cycle model. J Polit Econ 102(4):52-61

Chow GC (1985) A model of Chinese national income determination. J Polit Econ 93(4):782-792

Dassios I, Kontzalis C (2012) On the stability of equilibrium for a foreign trade model. In: Proceedings of the 32nd IASTED international conference

Dassios I, Kontzalis C, Kalogeropoulos G (2012) A stability result on a reformulated Samuelson economical model. In: Proceedings of the 32nd IASTED international conference

Dassios I, Kalogeropoulos G (2014) On the stability of equilibrium for a reformulated foreign trade model of three countries. J Ind Eng Int 10:71. doi:10.1007/s40092-014-0071-9

Dassios I, Zimbidis A (2014) The classical Samuelson's model in a multi-country context under a delayed framework with interaction. Dyn Contin Discrete Impuls Syst, Ser B, Appl Algorithms 21(4-5b):261-274

Day R (1999) Complex economic dynamics: an introduction to macroeconomic dynamics, vol 2. MIT Press, Cambridge

Dorf RC (1983) Modern control systems, 3rd edn. Addison-Wesley, Reading

Kuo BC (1996) Automatic control systems, 5th edn. Prentice Hall, New York

Ogata K (1987) Discrete time control systems. Prentice Hall, New York

Puu T, Gardini L, Sushko I (2004) A Hicksian multiplier-accelerator model with floor determined by capital stock. J Econ Behav Organ 56:331-348

Rosser JB (2000) From catastrophe to chaos: a general theory of economic discontinuities. Academic Publishers, Boston

Samuelson P (1939) Interactions between the multiplier analysis and the principle of acceleration. Rev Econ Stat 21:75-78

Westerhoff FH (2006) Samuelson's multiplier-accelerator model revisited. Appl Econ Lett 56:86-92

Wincoop E (1996) A multi-country real business cycle model. Scand J Econ 23:233-251 